\title{
Study on the antioxidant activity of lignin and its application performance in SBS elastomer
}

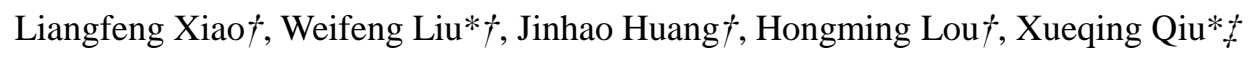

† School of Chemistry and Chemical Engineering, Guangdong Engineering Research Center for

Green Fine Chemicals, South China University of Technology, Wushan Road 381, Guangzhou,

Guangdong, 510640, China

\# School of Chemical Engineering and Light Industry, Guangdong University of Technology,

Waihuan Xi Road 100, Guangzhou Higher Education Mega Center, Guangzhou, 510006, P.R

China

*Corresponding authors

E-mail: weifengliu@ scut.edu.cn (W. Liu); cexqqiu@scut.edu.cn_(X. Q. Qiu).

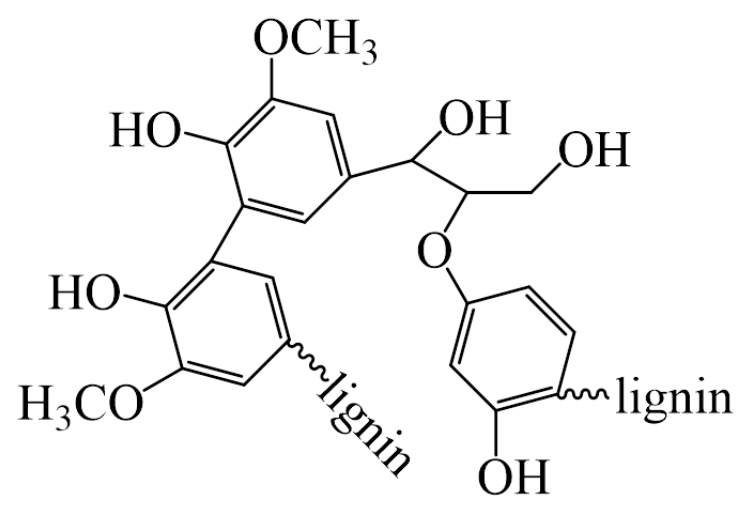

Figure S1. The structure diagram of lignin base unit. 

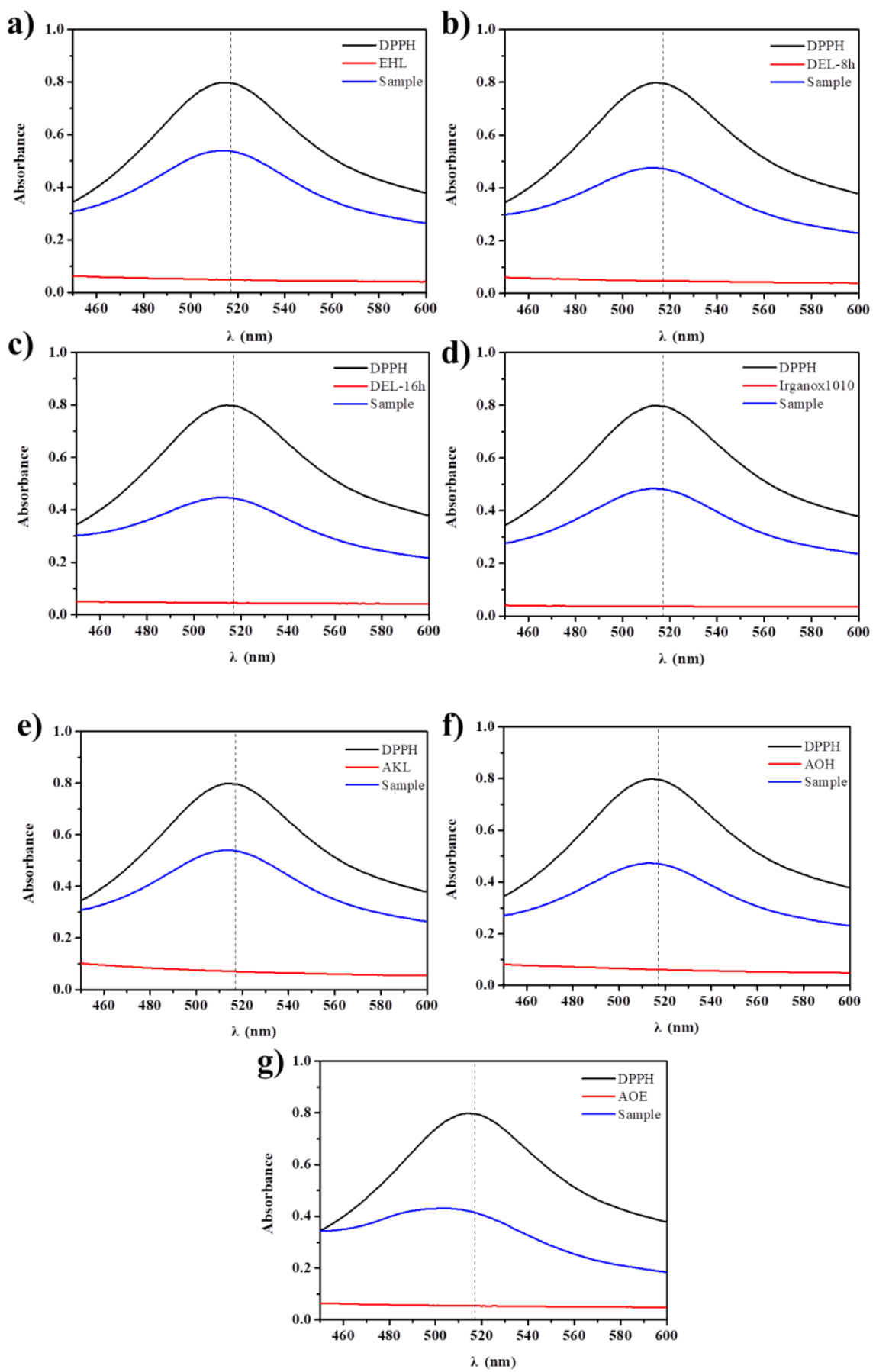

Figure S2. UV spectrum of a) EHL, b) DEL-8h, c) DEL-16h, d) Irganox1010, e) AKL, f) AOH and g) $\mathrm{AOE}$ at the concentration of $0.022 \mathrm{mg} / \mathrm{ml}$, Irganox1010 was compared as a reference. (Sample represents the mixed solution of lignin and DPPH) 

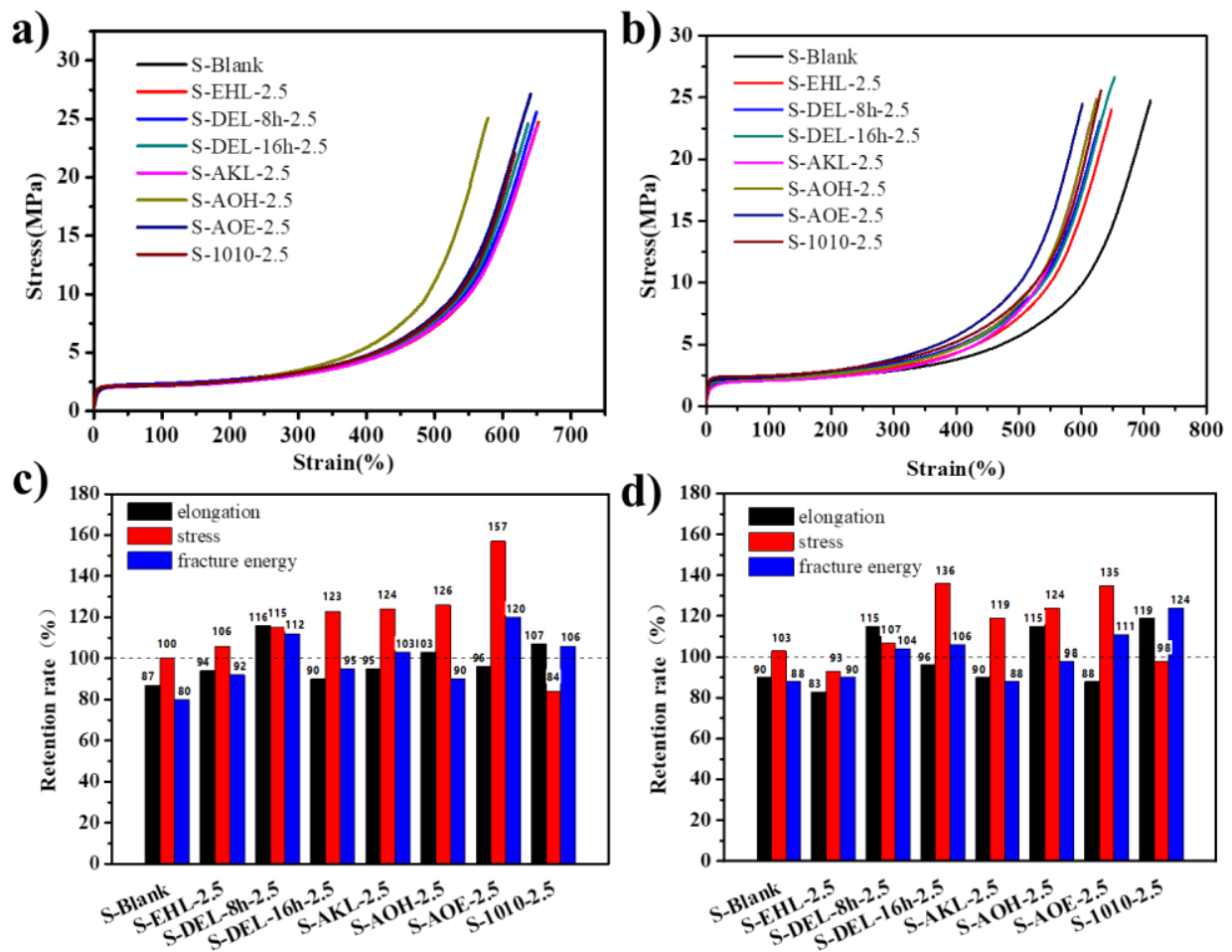

Figure S3. Mechanical properties of lignin/SBS composites: Engineering tensile stress-strain curves after aging for a) 3 days and b) 6 days, the retention ratios after aging for c) 3 days and d) 6 days.

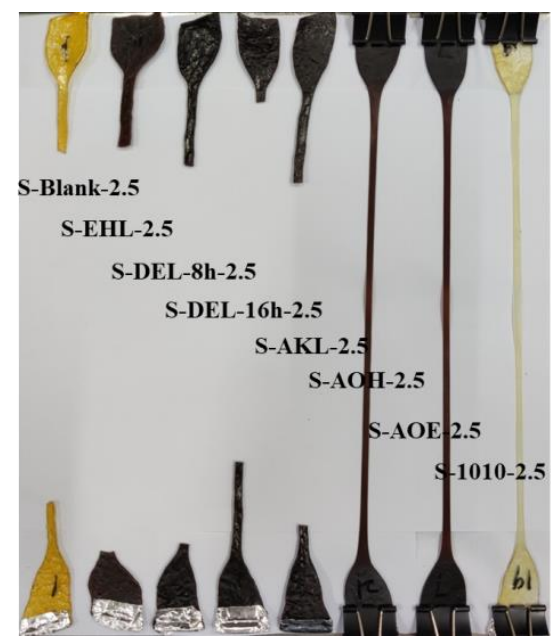

Figure S4. Lignin/SBS composites after 30 days of thermal oxygen aging. 
Table S1 The mechanical properties before and after aging for 3 or 6 days of lignin/SBS composites.

\begin{tabular}{|c|c|c|c|c|c|c|c|c|c|c|c|c|}
\hline \multirow{3}{*}{ sample } & \multicolumn{3}{|c|}{$\begin{array}{l}\text { Elongation at break } \\
\qquad(\%)\end{array}$} & \multirow{3}{*}{$\begin{array}{l}\text { Et } \\
(\%)\end{array}$} & \multicolumn{3}{|c|}{$\begin{array}{l}\text { Tensile strength } \\
\text { (MPa) }\end{array}$} & \multicolumn{4}{|c|}{$\begin{array}{l}\text { Fracture energy } \\
\qquad\left(\mathrm{MJ} / \mathrm{m}^{3}\right)\end{array}$} & \multirow{3}{*}{$\begin{array}{l}\text { Rt } \\
(\%)\end{array}$} \\
\hline & \multirow[b]{2}{*}{ before } & after & after & & & after & after & $(\%)$ & & after & after & \\
\hline & & $\begin{array}{c}3 \\
\text { days }\end{array}$ & $\begin{array}{c}6 \\
\text { days }\end{array}$ & & before & $\begin{array}{c}3 \\
\text { days }\end{array}$ & $\begin{array}{c}6 \\
\text { days }\end{array}$ & & before & $\begin{array}{c}3 \\
\text { days }\end{array}$ & $\begin{array}{c}6 \\
\text { days }\end{array}$ & \\
\hline S-Blank & $\begin{array}{l}746 \\
( \pm 3)\end{array}$ & $\begin{array}{l}650 \\
( \pm 2)\end{array}$ & $\begin{array}{r}675 \\
( \pm 36)\end{array}$ & $87 / 90$ & $\begin{array}{r}23.3 \\
( \pm 0.5)\end{array}$ & $\begin{array}{r}24.1 \\
( \pm 1.0)\end{array}$ & $\begin{array}{r}23.9 \\
( \pm 3.5)\end{array}$ & $103 / 103$ & 45.71 & 36.60 & 40.02 & $80 / 88$ \\
\hline S-EHL-2.5 & $\begin{array}{r}693 \\
( \pm 10)\end{array}$ & $\begin{array}{l}651 \\
( \pm 1)\end{array}$ & $\begin{array}{r}574 \\
( \pm 70)\end{array}$ & $94 / 83$ & $\begin{array}{l}25.1 \\
( \pm 3)\end{array}$ & $\begin{array}{r}26.6 \\
( \pm 1.8)\end{array}$ & $\begin{array}{r}23.3 \\
( \pm 1.7)\end{array}$ & $106 / 93$ & 40.34 & 37.24 & 36.27 & $92 / 90$ \\
\hline S-DEL-8h-2.5 & $\begin{array}{l}563 \\
( \pm 1)\end{array}$ & $\begin{array}{r}651 \\
( \pm 13)\end{array}$ & $\begin{array}{r}648 \\
( \pm 17)\end{array}$ & $116 / 115$ & $\begin{array}{r}22.0 \\
( \pm 0.4)\end{array}$ & $\begin{array}{r}25.4 \\
( \pm 1.0)\end{array}$ & $\begin{array}{r}23.6 \\
( \pm 2.1)\end{array}$ & $115 / 107$ & 34.60 & 38.72 & 35.98 & $112 / 104$ \\
\hline S-DEL-16h-2.5 & $\begin{array}{r}699 \\
( \pm 10)\end{array}$ & $\begin{array}{l}632 \\
( \pm 7)\end{array}$ & $\begin{array}{r}668 \\
( \pm 19)\end{array}$ & $90 / 96$ & $\begin{array}{l}20.0 \\
( \pm 3)\end{array}$ & $\begin{array}{c}24.5 \\
( \pm 1.0)\end{array}$ & $\begin{array}{r}27.2 \\
( \pm 1.4)\end{array}$ & $123 / 136$ & 38.27 & 36.21 & 40.61 & $95 / 106$ \\
\hline S-AKL-2.5 & $\begin{array}{r}682 \\
( \pm 16)\end{array}$ & $\begin{array}{l}651 \\
( \pm 2)\end{array}$ & $\begin{array}{l}615 \\
( \pm 2)\end{array}$ & $95 / 90$ & $\begin{array}{r}19.4 \\
( \pm 0.2)\end{array}$ & $\begin{array}{c}24.1 \\
( \pm 1.0)\end{array}$ & $\begin{array}{c}23.1 \\
( \pm 0.1)\end{array}$ & $124 / 119$ & 35.59 & 36.60 & 31.17 & $103 / 88$ \\
\hline S-AOH-2.5 & $\begin{array}{l}560 \\
( \pm 8)\end{array}$ & $\begin{array}{l}575 \\
( \pm 4)\end{array}$ & $\begin{array}{c}644 \\
( \pm 20)\end{array}$ & $103 / 115$ & $\begin{array}{r}21.0 \\
( \pm 0.1)\end{array}$ & $\begin{array}{c}26.4 \\
( \pm 1.4)\end{array}$ & $\begin{array}{c}26.1 \\
( \pm 1.2)\end{array}$ & $126 / 124$ & 36.43 & 32.90 & 35.78 & $90 / 98$ \\
\hline S-AOE-2.5 & $\begin{array}{l}680 \\
( \pm 1)\end{array}$ & $\begin{array}{l}651 \\
( \pm 1)\end{array}$ & $\begin{array}{c}601 \\
( \pm 13)\end{array}$ & $96 / 88$ & $\begin{array}{c}18.1 \\
( \pm 1.0)\end{array}$ & $\begin{array}{r}28.5 \\
( \pm 0.6)\end{array}$ & $\begin{array}{c}24.5 \\
( \pm 2.6)\end{array}$ & $157 / 135$ & 32.65 & 39.33 & 36.34 & $120 / 111$ \\
\hline S-1010-2.5 & $\begin{array}{l}579 \\
( \pm 5)\end{array}$ & $\begin{array}{l}621 \\
( \pm 6)\end{array}$ & $\begin{array}{r}691 \\
( \pm 13)\end{array}$ & $107 / 119$ & $\begin{array}{c}26.4 \\
( \pm 0.3)\end{array}$ & $\begin{array}{c}22.1 \\
( \pm 2.8)\end{array}$ & $\begin{array}{c}26.0 \\
( \pm 0.4)\end{array}$ & $84 / 98$ & 31.08 & 32.87 & 38.54 & $106 / 124$ \\
\hline
\end{tabular}

Table S2 Thermogravimetric data sheet of SBS, lignin/SBS composites and Irganox 1010/SBS composite, respectively.

\begin{tabular}{cccc}
\hline Sample & $\mathrm{T}_{5 \%}\left({ }^{\circ} \mathrm{C}\right)$ & $\mathrm{T}_{50 \%}\left({ }^{\circ} \mathrm{C}\right)$ & $\mathrm{T}_{\max }\left({ }^{\circ} \mathrm{C}\right)$ \\
\hline S-Blank & 383.4 & 437.3 & 435.6 \\
S-EHL-2.5 & 373.4 & 435.6 & 434.2 \\
S-DEL-8h-2.5 & 378.4 & 437.6 & 435.3 \\
S-DEL-16h-2.5 & 380.8 & 437.3 & 435.2 \\
S-AKL-2.5 & 375.9 & 437.5 & 435.7 \\
S-AOH-2.5 & 375.2 & 436.6 & 435.0 \\
S-AOE-2.5 & 374.7 & 435.4 & 434.7 \\
S-1010-2.5 & 381.9 & 437.3 & 435.4 \\
\hline
\end{tabular}

